\title{
La importancia de la relación entre las micorrizas arbusculares y el fósforo en nuestros suelos
}

\author{
Paula Gadea ${ }^{1}$ \& Wagner Peña ${ }^{1,2}$ \\ 1. Protección de cultivos de la Maestría PPCARN en Ciencias Agrícolas y Recursos Naturales, Universidad \\ de Costa Rica. \\ 2. Encargado Cátedra de Gestión Sostenible del Suelo, ECEN, UNED: wpena@uned.ac.cr
}

Recibido: 12 noviembre 2012

Aceptado: 16 enero 2013

\begin{abstract}
RESUMEN
La agricultura de hoy en día requiere de tecnología limpia y de mayores conocimientos en el campo de la sostenibilidad y protección del medio ambiente. El abuso de fertilizantes, sobre todo fosfatados, lleva al deterioro de los agroecosistemas, por lo que se busca reutilizar los recursos para sustituir o complementar los sistemas agrícolas existentes. Tal es el caso del conocimiento en microorganismos beneficiosos para la agricultura, como las micorrizas arbusculares (VAM). Estos agentes biológicos contribuyen en la solubilización de elementos esenciales para los cultivos, como lo son varios oligoelementos y, especialmente, el fósforo en el suelo. En este último tema es muy importante para la fertilidad de los suelos en Costa Rica, ya que la mayoría de los suelos presentan poca disponibilidad del elemento y es necesario alternativas sostenibles que sustituyan el aporte de sales fertilizantes fosfatadas.

El trabajo pretende analizar información sobre las micorrizas VAM y el uso en la agricultura actual, sobre todo como biofertilizante y como una alternativa más para pequeños agricultores costarricenses.
\end{abstract}

Palabras clave: Sostenibilidad, suelos, microbiología, VAM.

\footnotetext{
ABSTRACT

Farming today requires clean technology and a greater knowledge of sustainability and environmental protection. Overuse of fertilizers, especially phosphate, leads to the deterioration of agroecosystems; thus, we need to re-use resources in order to replace or supplement existing farming systems. One example is the case of microorganisms that are beneficial in agriculture, such as arbuscular mycorrhizae (AMF). These biological agents contribute to the solubilization of elements that are essential for crops, such as multiple trace elements and, particularly, phosphorus in the soil. Phosphorus is very important for soil fertility in Costa Rica, where most soils have limited availability and need sustainable alternatives to replace the supply of phosphatic fertilizer salts.
}

This paper analyzes information on VAM and their use in agriculture today, particularly as biofertilizer and as an alternative for small farmers in Costa Rica.

Key words: Sustainability, soil microbiology, AMF.

\section{Introducción}

\section{Las micorrizas}

Según algunos autores y por experiencias en la agricultura práctica, las micorrizas son organismos microscópicos de tipo fúngico beneficioso, que se definen como una asociación simbiótica entre las raíces de ciertas plantas y hongos del suelo, las cuales permiten el intercambio de sustancias nutritivas, metabolitos esenciales y sustancias hormonales (Triana 2004, Rivera et al. 2003; Blanco y Salas, 1997). Es por ello que se les encuentra comúnmente en la zona radicular de una mayoría de plantas frutales, arvenses y hortícola, entre otras, aspecto que es muy importante en cultivos de exportación en regiones tropicales como Costa Rica.

Entre los principales beneficios que recibe una planta colonizada por hongos micorrizógenos se destacan los siguientes:

- Mayor crecimiento de las plantas principalmente en suelos con bajo contenido de nutrientes.

- Mayor capacidad de absorción de nutrientes poco móviles del suelo $(\mathrm{P}, \mathrm{Zn}, \mathrm{Cu}$, principalmente).

- Mayor capacidad de absorción de agua y tolerancia a la sequía.

- Protección contra patógenos de la raíz. 
- Detoxificación de metales pesados (Triana 2004, Guerrero 1996).

Sieverding (1991) clasifica este tipo de microorganismo del suelo con base en su estructura, morfología y modo de infección, en dos tipos principales: ectomicorrizas y endomicorrizas; este último grupo se divide en varios subtipos, como lo son las ectendomicorriza, arbutoides, monotropoides, ericoides, orquidáceas y las arbusculares, que son las más comunes (Blanco y Salas, 1997).

Las micorrizas vesículo arbusculares, llamadas por sus siglas en inglés VAM, deben su nombre a la formación de vesículas (Fig.1a) y arbúsculos (Fig.1b) lugar donde se lleva a cabo el intercambio de sustancias, estas pertenecen al orden Glomales; el suborden Glominaeae tiene dos familias: Glomaceae que comprende los géneros Glomus y Sclerocystis, y Acaulosporaceae que incluye dos géneros: Acaulospora $\mathrm{y}$ Entrophospora. El suborden Gigasporineae tiene una sola familia: Gigasporeaceae con dos géneros: Gigaspora y Scutellospora (Bentivenga y Morton 1994, citados por Blanco y Salas 1997).

Según Rivera et al. (2003) el principal elemento durante el establecimiento del hongo en la raíz es el fósforo $(\mathrm{P})$, ya que las hifas absorben el "P inorgánico" del suelo a través de un proceso activo, convirtiéndose luego de un proceso de fosforilación en gránulos de polifosfato (2P) (Fig. 2). Luego son transportados por la corriente citoplasmática hasta las vesículas, donde pueden ser almacenados temporalmente o ir directamente hacia los arbúsculos. Cabe mencionar que el fósforo es un macroelemento del suelo muy poco móvil, por lo general queda adsorbido en las arcillas o formando complejos orgánico-mineral.

Dentro de la matriz arbuscular, el polifosfato es hidrolizado por el complejo enzimático fosfatasa alcalina en fosfato inorgánico $(\mathrm{Pi}) \mathrm{y}$ transferido a la célula vegetal, pasando a través de la interfase hongo - planta. Este proceso de transporte en la interfase está mediado por los cambios de potencial de membrana, tanto de la planta como del micelio y del sistema de transporte ATPasa - bomba de protón (Gianinazzi
- Pearson y Gianinazzi 1983, Dexheimer et al. 1986, citados por Rivera et al. 2003).

Simultáneamente, en el sentido contrario ocurre la transferencia de carbohidratos provenientes de la fotosíntesis vía floema. Llegan a la célula vegetal en forma de sacarosa la cual es hidrolizada a través de una enzima de tipo invertasa, convertida en dos monómeros, que a su vez se descomponen posteriormente y se fosforilan en moléculas de triosa - P, para ser transferidos al simbionte vía arbúsculos (Letacon y Obatón 1983, Smith et al. 1994, citados por Rivera et al. 2003).

Generalmente se desconocen los mecanismos químicos del hongo VAM involucrados en la absorción de este elemento esencial, no obstante se sabe que se toma en forma de ión ortofosfato $\left(\mathrm{P}_{2} \mathrm{O}_{5}\right)$ y se transporta a través de las hifas del hongo en forma de polifosfato a una velocidad de $2 \times 10^{-9} \mathrm{~mol} \cdot \mathrm{cm}^{-2}$ (Rivera et al., 2003).
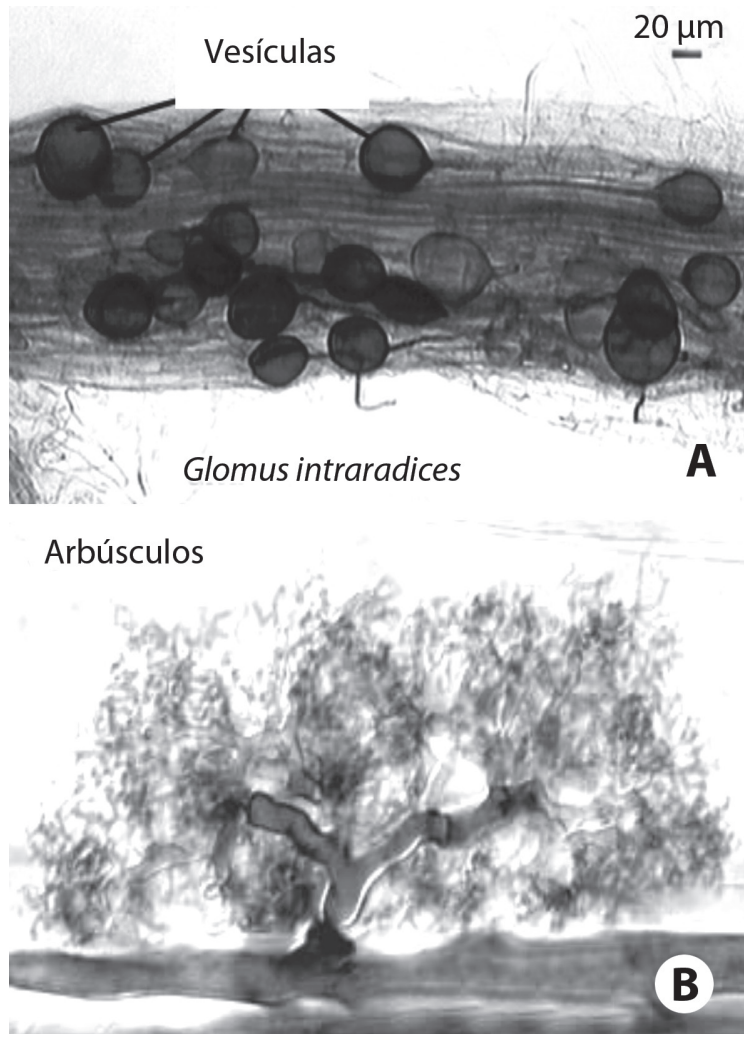

Figura 1. Raíz con vesículas (A) y arbúsculos (B). Imágenes cortesía de Eduardo Salas (UNA). 


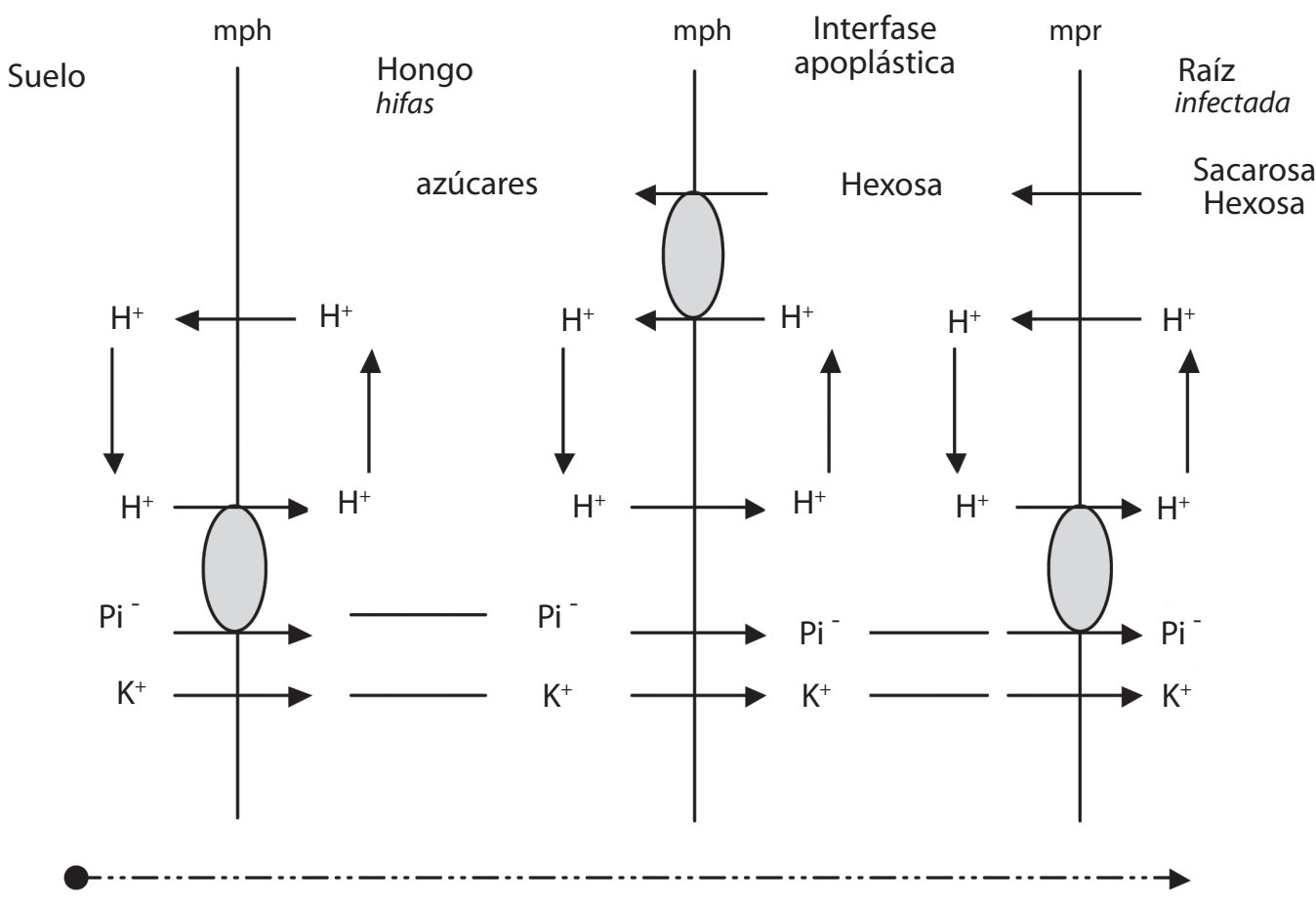

Figura 2. Posible mecanismo de transferencia de fosfato vía interfase micorrízica $\mathrm{mph}$ y $\mathrm{mpr}=$ membranas plasmáticas del hongo y de la raíz respectivamente (adaptado de Rivera et al. 2003) $\mathrm{Pi}^{-}=\mathrm{P}_{2} \mathrm{O}_{5}$

Como se mencionó anteriormente, los hongos micorrizógenos estimulan el crecimiento de la planta mediante un aumento en la capacidad de toma de nutrientes, particularmente el fósforo, debido a incrementos de varios factores: la superficie de absorción y exploración del volumen del suelo por el micelio extramatrical, el diámetro de la hifa pequeña principal aumenta el área de absorción de $\mathrm{P}$ en comparación con raíces no micorrizadas, también se favorece el flujo de $\mathrm{P}$ por unidad de superficie, la producción de ácidos orgánicos y fosfatasas que catalizan la liberación de $\mathrm{P}$ de los complejos orgánicos y la formación de polifosfatos por los hongos micorrizógenos (Marschner y Dell 1994, citados por Bucking 2003).

Es así que en la infección micorrizal, la eficiencia del $\mathrm{P}$ en la nutrición de las plantas hospederas no es debido a la taza de absorción de este elemento cuando es suplido, pero la capacidad de acumulación del P cuando es dado externamente en altas cantidades y su movilización para almacenarlo bajo condiciones de estrés mantiene un continuo flujo de $\mathrm{P}$ de la micorriza al hospedero, aún en condiciones severas, aspecto que resulta significativo en cultivos tropicales del país.

Es importante señalar que la infección de las micorrizas y el crecimiento de las plantas se ve disminuida en condiciones de altas cantidades de $\mathrm{P}$ soluble, sobre todo provenientes de sales fosfatadas; esto se debe a que se presenta un alto costo de carbón por parte de la planta hospedera, ya que alrededor de un $20 \%$ del carbón asimilado por la planta es traslocado al hongo simbiótico en la asociación.

Biemsa (s.f.) afirma que concentraciones elevadas de nitrógeno y fósforo inhiben la colonización de las raíces por parte de las micorrizas, a la vez recomiendan reducir los aportes de fertilizantes hasta después de la colonización, o utilizar fertilizantes NPK cuyo P sea inferior a 7. Concentraciones inferiores a $110 \mathrm{ppm}$ de $\mathrm{N} \mathrm{y}$ 80 ppm de $\mathrm{P}$ en el suelo son favorables para la colonización de las micorrizas, mientras que las 
concentraciones óptimas de estos elementos son respectivamente 60 y $50 \mathrm{ppm}$.

\section{Interacción micorrizas-fósforo y \\ microorganismos solubilizadores}

Varios autores concuerdan que la absorción de $\mathrm{P}$ en plantas micorrizadas se favorece en presencia de microorganismos solubilizadores de fósforo (Rivera et al. 2003, Ravnskov y Jakobsen 1999, Singh y Kapoor 1998, Singh y Singh 1993).

Singh y Kapoor (1998) efectuaron un ensayo donde combinaron microorganismos solubilizadores de fósforo (Bacillus circulans y Cladosporium herbarum) con la micorriza Glomus fasciculatum en garbanzo (Vigna radia$t a$ ), en presencia y ausencia de roca fosfórica. Este cultivo es cada vez más utilizado en zonas de recuperación de suelos, como en ultisoles de la zona norte de Costa Rica. En el ensayo determinaron que la concentración de $\mathrm{P}$ en la biomasa de la planta fue mayor en presencia de los microorganismos solubilizadores de fósforo (MSP); además, la inoculación con MSP y la micorriza aumentó significativamente la concentración de $\mathrm{P}$ en la biomasa de la planta, a pesar de la ausencia de la roca fosfórica. Sin embargo, en presencia de la roca fosfórica, la concentración de $\mathrm{P}$ aumentó sólo en los tratamientos que recibieron $G$. fasciculatum o la combinación de ésta con $C$. herbarum, eso puede deberse a que se promueve una mejor utilización del $\mathrm{P}$ proveniente del banco de $\mathrm{P}$ nativo en el suelo así como de la roca fosfórica y la acción de los MSP y la G.fasciculatum.

La inoculación con B.circulans o C.herbarum con o sin G.fasciculatum incrementó la concentración de $\mathrm{P}$ tanto en el grano y como en la paja del garbanzo, comparado con los controles no inoculados. Los autores concluyen que la toma de $\mathrm{P}$ es mayor cuando al suelo se le añaden enmiendas de MPS ya que se da una mejor absorción de $\mathrm{P}$ por parte de la micorriza (Singh y Kapoor 1998).

En un estudio realizado en soya, Singh y Singh (1993) probaron la interacción de la roca fosfórica con Bradyrhizobium japonicum, micorrizas vesículo arbusculares y varios MSP (Pseudomonas striata, Bacillus polymyxa y Aspergillus awamori). Informan que, en general la aplicación de roca fosfórica, Bradyrhizobium, micorrizas y los MSP en combinación de 2 o 3 de ellos resultó en incrementos en la nodulación, el crecimiento de la planta, de la cosecha de grano y la toma de N y P. Los autores reportan que la mayor toma de $\mathrm{P}$ por los granos de soya se mostró con la mezcla de micorrizas, Bradyrhizobium y A. awamori, en ausencia de la roca fosfórica. También mencionan que la cosecha del grano y de la paja no se aumentó por la adición de roca fosfórica o la inoculación con micorrizas, pero que se incrementó después de la inoculación con Bradyrhizobium o MSP.

Otros autores notaron que en cucurbitáceas Pseudomonas fluorescens DF57, tiene un efecto simbiótico positivo en el crecimiento y asimilación de $\mathrm{P}$ en las plantas tratadas con dos micorrizas arbusculares (Glomus intraradices y G. caledonium), señalan que el contenido total de $\mathrm{P}$ fue mayor en plantas que crecieron en simbiosis con $G$. intraradices que en las de otros tratamientos, sin embargo G. caledonium y $P$. fluorescens DF57 mostraron un efecto sinergístico en donde las plantas inoculadas con G.caledonium presentaron un contenido superior de $\mathrm{P}$ que las plantas testigo en ausencia de $P$. fluorescens DF57 (Ravnskov y Jakobsen, 1999).

Por otro lado autores como Meyer y Linderman (1986), Orozco et al. (1986) y Ames y Bethlenfalvay (1984) citados por Rivera et al. (2003), plantean que determinados grupos funcionales de microorganismos asociados a la hifosfera del hongo pueden aumentar indirectamente la solubilización del fosfato mediante la activación del complejo enzimático de las fosfatasas.

Ensayos realizados por Piccini y Azcón (1987) y Piccini et al. (1988) citados por Rivera et al. (2003), evidenciaron en alfalfa el papel de la asociación entre microorganismos solubilizadores de $\mathrm{P}$ y hongos formadores de micorrizas vesículo arbusculares. 
Interacción micorrizas-fósforo y resistencia a plagas y enfermedades

Las micorrizas arbusculares han sido reportadas por tener efecto supresivo contra enfermedades causadas por hongos patógenos que atacan raíces (Dehne 1982, Garbaye 1991, Sharma et al. 1992, citados por Bodker et al. 1998). Muchos mecanismos están envueltos en las interacciones entre las micorrizas y los patógenos de suelo, pero el trasfondo de la supresión a las enfermedades aun no se comprende en su totalidad. (Linderman 1994, Azcon-Aguilar y Barea 1996, Cordier et al. 1996, Dumas-Gaudot et al. 1996, Trotta et. al 1996, citados por Bodker et al. 1998). Este tipo de micorrizas se reconoce por su capacidad de tomar P y otros nutrientes, esta superioridad nutricional ha sido el mecanismo propuesto como la reducción de enfermedades radiculares (Linderman 1994, citado por Bodker et al. 1998).

A pesar de lo anterior reportes del rol del fósforo en la resistencia del hospedero han sido objetados. Trotta et al. (1996) citados por Bodker et al. (1998), estudiaron el efecto de la interacción de $\mathrm{P}$ y Phytophtora parasitica con micorrizas arbusculares en tomate y demostraron que el efecto protector de la micorriza no esta relacionado con el estatus nutricional del hospedero.

En una investigación realizada por Bodker et al. (1998), se evalúo el efecto de niveles de $\mathrm{P}$ en plantas de arveja (Pisum sativum) inoculadas con G.intraradices para determinar el potencial de control contra Aphanomyces euteiches (patógeno radicular que causa severos problemas). Concluyen que las plantas inoculadas con el hongo G.intraradices incrementaron la toma de $\mathrm{P}$ y que la concentración de $\mathrm{P}$ en la planta disminuyó el desarrollo de la enfermedad en arvejas. Notaron una reducción en la severidad de la enfermedad en el epicótilo de las plantas micorrizadas respecto a las no inoculadas, señalando que en las plantas con micorrizas se induce un factor sistémico responsable de la resistencia ante la enfermedad.

Por otro lado Carling et al. (1996), estudiaron el efecto individual y combinado, en vivero, de las micorrizas Gigaspora margarita y Glomus etunicatum, el nematodo Meloidogyne arenaria y cuatro dosis de fertilización fosfórica $(0,25$, 75 y 125 micro g/g suelo) en un suelo cultivado con maní. El mejor crecimiento y cosecha ocurrió a 75 o $125 \mathrm{~g} \mathrm{P}$ a pesar que, los tratamientos incluían inoculación micorrízica. La cosecha y el crecimiento en el suelo de maní fue estimulado por el desarrollo de las micorrizas, y el crecimiento de las plantas de maní solo fue suprimida por M.alernaria a 0 y 25 micro g P. En las inoculaciones las micorrizas incrementaron la tolerancia de la planta al nematodo y contrarrestó las reducciones en crecimiento causadas por éste a los dos niveles más bajos de $\mathrm{P}$.

\section{Estudios de caso}

A continuación se presentan resultados obtenidos en varios cultivos donde se contempla la interacción micorrizas arbusculares y fósforo.

Café

Estudios de los efectos de la inoculación de micorrizas en café tanto en campo como en laboratorio se realizan desde 1986 en Brasil, e indican que en suelos de baja fertilidad, la precolonización de las plantas de café antes de ser llevadas a campo incrementan la cosecha hasta en un 74\%; sin embargo el efecto se presenta si existe una cantidad adecuada de $\mathrm{P}$ disponible en el suelo (Siquiera et al. 1993 citados por Siquiera et al. 1998). Aunque esos resultados fueron alentadores, solo se estudiaron con una sola dosis de superfosfato.

Siquiera et al. (1998), investigó desde el año 1989 el alcance en el desarrollo y productividad de plantas de café preinoculadas, hasta un periodo de 6 años después del tratamiento con micorrizas arbusculares, en un suelo de baja fertilidad y con cinco dosis de $\mathrm{P}_{2} \mathrm{O}_{5}(0,20,40$, 80 y $160 \mathrm{~g} /$ planta) adicionado al transplante. Las dosis de fertilizante fueron combinadas con 7 tratamientos de hongos (control sin micorrizas, precolonización con una mezcla de Glomus clarum y Gigaspora margarita y 5 aislamientos 
de Glomus etunicatum). Entre los resultados obtenidos se encuentran:

- El peso de las plantas y el diámetro de tallos se incrementaron considerablemente con la aplicación de $\mathrm{P}$ y fue mayor en las tratadas con micorrizas que en los controles, 19 meses después del transplante (MDT), pero no hubo diferencia a los 26 MDT.

- La colonización del sistema radical y el número de esporas se redujo en presencia de las dosis más altas de P, 26 MDT.

- La primera cosecha se incrementó considerablemente por la interacción del $\mathrm{P}$ y los tratamientos con precolonización (38\% de aumento sobre el control) 26 MDT.

- Los aislamientos de G. etunicatum mostraron aumentos en la cosecha superiores al $50 \%$. La dosis de fertilizante que logró la máxima producción fue de $207 \mathrm{~g} /$ planta de $\mathrm{P}_{2} \mathrm{O}_{5}$ para las plantas no precolonizadas y de $100 \mathrm{~g} /$ planta para las precolonizadas a los 26 MDT.

- En 1992, 1993 y 1995 la cosecha fue afectada por la dosis de fertilizante pero no por la inoculación de micorrizas. En 1994, el P versus la interacción de Gigaspora margarita y las variedades de G. etunicatum, presentaron cosechas mayores que el testigo.

- Considerando la producción acumulada en 5 años, la aplicación de $\mathrm{P}$ incrementó la cosecha en todos los tratamientos, mientras que en la precolonización los efectos fueron disminuidos, a pesar de la inconsistencia en los tratamientos de micorrizas, los efectos de la precolonización fueron detectados a la quinta cosecha en algunos tratamientos.

- Basados en el total de los 5 años de cosechas, la máxima productividad en los tratamientos con micorrizas fue en los tratados con Gigaspora margarita a $20 \mathrm{~g} / \mathrm{planta}$ con $\mathrm{P}_{2} \mathrm{O}_{5}$ y Gigaspora margarita y $G$. etunicatum a la mayor dosis ( $160 \mathrm{~g} /$ planta de $\left.\mathrm{P}_{2} \mathrm{O}_{5}\right)$.

- La disminución el efecto micorrízico a través del tiempo esta relacionado con la colonización de las plantas no precolonizadas por parte de los hongos micorrizógenos nativos. Rivera et al. 2003, también señala que es de vital interés considerar la cantidad de propágulos nativos cuando se trabaja en experimentos con micorrizas en suelos no esterilizados.

- En conclusión se determinó que una adecuada dosis de $\mathrm{P}$ aplicada al transplante, la precolonización de las plantas y la selección de un hongo micorrízico arbuscular adecuado, incrementa el desarrollo del cultivo en etapas tempranas y la productividad del café en suelos de baja fertilidad en Brasil.

\section{Soya y sorgo}

Pacovsky et al. (1986) citados por Rivera et al. (2003) realizaron estudios con plantas de soya (Glicine max.L) y sorgo (Sorghum vulgare) inoculadas con distintas especies de hongos micorrizógenos y sin inoculación, con aplicación de dosis crecientes de fertilizante fosfórico. En las plantas no inoculadas el contenido del elemento se incrementó exponencialmente con el aumento en las dosis de $\mathrm{P}$, sin embargo en las micorrizadas el incremento fue logarítmico siguiendo el propio desarrollo fúngico. Además, se pudo afirmar que las plantas inoculadas y las tratadas sólo con fertilización fosfórica difieren anatómicamente y fisiológicamente entre sí, sin embargo utilizan la misma fuente de fosfatos solubles del suelo.

\section{Maíz}

Asmah (1995) investigó el efecto de dos fuentes de $\mathrm{P}$ (tripe superfosfato y roca fosfórica) con dosis equivalentes a $44 \mathrm{~kg} / \mathrm{ha}$ y $22 \mathrm{~kg} / \mathrm{ha}$, en plantas de maíz inoculadas con micorrizas vesículo arbusculares sembradas en maceteros. Se midió la cantidad de materia seca en la cosecha, el contenido de nutrientes y la infección radicular en un suelo oxisol y otro alfisol.

La aplicación de $44 \mathrm{~kg} / \mathrm{ha}$ respecto a las plantas a las cuales no se les aplicó P, resultó estadísticamente no significativa en la variable de infección radical, pero fue significativamente superior cuando se aplicó en $\mathrm{P}$ en forma de superfosfato a la dosis de $22 \mathrm{~kg} /$ planta. Los tratamientos con roca fosfórica, a ambas dosis, 
resultaron ser significativamente mejores en el comportamiento de infección radical que los controles sin $\mathrm{P}$ o cuando el triple superfosfato fue aplicado a $44 \mathrm{~kg} / \mathrm{h}$. La asimilación de $\mathrm{P}$ en la planta fue superior en ambos suelos con los diferentes tratamientos de $\mathrm{P}$ comparados con las plantas testigo (Asmah 1995).

En relación al contenido de nutrientes Asmah (1995), reporta que no se mostró un efecto de los tratamientos en el contenido de $\mathrm{Al}$ y $\mathrm{Zn}$. En el suelo alfisol, la absorción de Mn se redujo debido al incremento de la infección de las plantas tratadas con superfosfato. El mayor contenido de Mn también fue encontrado en plantas fertilizadas con la dosis mas alta de superfosfato en relación a las tratadas con roca fosfórica, sin embarbo la infección micorrízica de las raíces con esta última fuente fue superior.

Con la excepción de las plantas tratadas con roca fosfórica a razón de $22 \mathrm{~kg} / \mathrm{ha}$, la materia seca de las plantas con todas las fuentes de $\mathrm{P}$ fue significativamente superior que los controles (Asmah 1995).

Por otro lado, Páez (2006) menciona que la eficiencia del $\mathrm{N}$ y del $\mathrm{P}$ en el cultivo de maíz, se incrementó debido a la actividad de micorrizas en el suelo.

Una investigación realizada por Polo (2005), se informa que plantas de maíz asociadas a micorrizas del género Glomus presentaron mayores índices de crecimiento, expresados en biomasa (materia seca y área foliar) debido a una mejor nutrición mineral por parte del hospedero, especialmente el P. Este mayor crecimiento de plantas micorrizadas está relacionado con mayores concentraciones de nutrientes $(\mathrm{N}, \mathrm{P}, \mathrm{K}, \mathrm{Ca}, \mathrm{Mg}$, $\mathrm{S})$ y azúcares en tejido, debido al rol del $\mathrm{P}$ en la síntesis de carbohidratos en plantas. Igualmente, plantas micorrizadas aumentan la concentración de proteínas foliares con respecto a no micorrizadas con niveles bajos y medios de $\mathrm{P}$ disponible (1 y $40 \mathrm{mg} / \mathrm{kg}$ ). Los resultados permiten suponer que la asociación simbiótica con Glomus sp contribuye con la síntesis de proteínas de estrés, por déficit de $\mathrm{P}$ en el suelo. El estrés conduce a la expresión diferencial de la información genética, produciendo cambios en la síntesis de nuevas proteínas, llamadas micorrizinas, las cuales posiblemente dotan a las plantas con la capacidad de adaptarse al estrés medioambiental por medio de ajustes fisiológicos y bioquímicos.

\section{Cebolla}

La cantidad de $\mathrm{P}$ en el tejido de las plantas y en la solución del suelo tiene influencia en la colonización del suelo por parte de las micorrizas arbusculares (Sanders 1975, Menge et al. 1978, Tawaraya et al. 1994, citados por Tawaraya et al. 1998). La nutrición de la planta hospedero afecta tanto la cantidad como la calidad de los exudados radicales. La deficiencia de $\mathrm{P}$ en las plantas incrementa los exudados de amino ácidos, azúcares, ácidos orgánicos (Ratnayake et al. 1978, Graham et al. 1981, Schwab et al. 1983, citados por Tawaraya et al. 1998), y en los exudados radicales en plantas de Citrus sp, trébol, cebolla y zanahoria deficientes en $\mathrm{P}$ (Tawaraya et al. 1998), y también se ha demostrado un incremento en el crecimiento de hifas en micorrizas vesículo arbusculares bajo esta condición. Además, en Gigaspora margarita, se aumenta la formación de apresorios en deficiencia de este elemento (Tawaraya et al. 1998); éste autor indica que el mecanismo de cómo los componentes de los exudados radicales afectan la colonización, aún no son bien conocidos.

Producto de dichas investigaciones (Tawaraya et al. 1998) realizaron un ensayo donde estudiaron el efecto de los exudados radicales en plantas de cebolla con deficiencia de $\mathrm{P}$ y en condiciones óptimas del elemento en la formación de apresorios y colonización de G.margarita. Llegaron a la conclusión que después de 24 días de inoculación, los compuestos hidrofóbicos encontrados en los exudados radiculares de plantas deficientes en $\mathrm{P}$, incrementaron la formación de apresorios y por tanto favorecieron el desarrollo de la micorriza.

\section{Bambú}

La fertilización con $\mathrm{P}$ es un factor importante para el crecimiento de plantas de bambú (Dendrocalamus asper); la aplicación de mezclas de inóculos de micorrizas han demostrado 
tener un significativo crecimiento de plantas de bambú en vivero (Verma y Jamaluddin 1994, citados por Verma y Arya 1998).

Plantas micropropagadas de bambú son altamente dependientes de las micorrizas arbusculares (Verma y Arya 1998), estos autores evaluaron diferentes aislamientos de micorrizas arbusculares y abono orgánico de estiércol en la biomasa y asimilación de $\mathrm{P}$ en plantas de cultivo in vitro, así como la producción de esporas de las micorrizas en la rizosfera en dos tipos de suelo.

Después de 12 meses, encontraron un efecto positivo de la inoculación en la concentración de $\mathrm{P}$ en los brotes, también en la colonización y producción de esporas en el medio constituido por arena-suelo. En el suelo con estiércol, la respuesta de esos parámetros fue mejor, sin embargo tuvo mejor influencia en la producción de esporas, altura y biomasa seca.

\section{Flores Dragón}

En el cultivo ornamental de flores de dragón (Antirrhinum majus L.), Besmer y Koide (1999) estudiaron el efecto del $\mathrm{P}$ y las micorrizas en la producción de etileno (compuesto tóxico que limita la vida poscosecha de las flores). La colonización de micorrizas no afectó el número total de flores por estaca ni la concentración de P en la flor, pero aumentó significativamente la vida pos-cosecha (tiempo en el florero) y disminuyó la producción de etileno. Con lo anterior se concluye que la colonización micorrízica puede ser una alternativa viable como inhibidor de la producción de etileno y compuestos tóxicos como el tiosulfato de plata.

El incremento en la concentración de P (15 versus 3 micro $\mathrm{g} \mathrm{P} / \mathrm{ml}$ ), aumentó el peso fresco y el número total de flores por estaca. En contraste con la colonización de las micorrizas, al aumentar la fertilización de P también se favoreció la producción de etileno. No se mostró una respuesta significativa de la fertilización fosfórica en relación a la vida en florero, por lo que sugieren que hay otros factores involucrados en la vida pos cosecha, sin embargo tratamientos con $\mathrm{P}$ en flores cortadas aumentó la producción de etileno. Aparentemente el $\mathrm{P}$ no contempla el efecto de las micorrizas, ya que la colonización de las micorrizas disminuyó la producción de etileno sin influenciar significativamente la concentración de $\mathrm{P}$ en las flores (Besmer y Koide 1999).

\section{Literatura citada}

Asmah, AE. 1995. Effect of phosphorus source and rate of application on VAM fungal infection and growth of maize (Zea mays L.) (disco compacto, $8 \mathrm{~mm}$ ). Mycorrhiza 5 (3): 223-228 Disponible en base de datos Teel, CD volumen 30 .

Carling, DE; Roncadori, RW; Hussey, RS. 1996. Interactions of arbuscular mycorrhizae, Meloidogyne arenaria, and phosphorus fertilization on peanut (disco compacto, $8 \mathrm{~mm}$ ). Mycorrhiza 6(1): 9-13. Disponible en base de datos Teel, CD volumen 30.

Besmer, YL ; Koide, RT. 1999. Effect of mycorrhizal colonization and phosphorus on ethylene production by snapdragon (Antirrhinum majus L.) flowers (disco compacto, $8 \mathrm{~mm}$ ) Mycorrhiza 9 (3):161-166. Disponible en base de datos Teel, CD volumen 291

Biemsa s.f. Ecofungi. s.n.t. Consultado el 05 nov. 2006. Disponible en www.biemsa.com

Blanco, FA; Salas EA. 1997. Micorrizas en la agricultura: contexto mundial e investigación realizada en Costa Rica. Agronomía Costarricense 21(1):55-67.

Bodker, L; Kjoller, R; Rosendahl, S. 1998. Effect of phosphate and the arbuscular mycorrhizal fungus Glomus intraradices on disease severity of root rot of peas (Pisum sativum) caused by Aphanomyces euteiches (disco compacto, $8 \mathrm{~mm}$ ) Mycorrhiza 8 (3): 169-174. Disponible en base de datos Teel, CD volumen 241 .

Bucking H, HW. 2003. Uptake and transfer of nutrients in ectomycorrhizal associations: interactions between photosynthesis and phosphate nutrition (disco compacto, $8 \mathrm{~mm}$ ). Mycorrhiza 13 (2):59-68. Disponible en base de datos Teel, CD volumen 414

Guerrero, E. 1996. Micorrizas: Recurso Biológico del Suelo. Editorial Fondo FEN. Bogota, Colombia. 208 p.

Páez, O. 2006. Las Micorrizas: Alternativa Ecológica para una Agricultura Sostenible. Consultado el 29 nov. 
2006. Disponible en http://www.soil-fertility.com/micorhize/espagnol/index.shtml

Polo, C. 2005. Mecanismos de adaptación de plantas de maíz en asocio con micorrizas arbusculares en suelos con baja disponibilidad de fósforo: Resúmenes de trabajos de grado de maestría. Universitas Scientiarum 10(1): 113-118. Consultado el 28 nov. 2006. Disponible en http://www.javeriana.edu.co/ciencias/ universitas/vol10n1/12-RESUMENES.pdf

Ravnskov, S; Jakobsen, I. 1999. Effects of Pseudomonas fluorescens DF57 on growth and P uptake of two arbuscular mycorrhizal fungi in symbiosis with cucumber (disco compacto, $8 \mathrm{~mm}$ ). Mycorrhiza 8 (6): 329-334. Disponible en base de datos Teel, CD volumen 291.

Rivera, R.A.; Fernández, F.; Hernández, A.; Martín, JR; Fernández, K. 2003. El manejo efectivo de la simbiosis micorrízica, una vía hacia la agricultura sostenible: Estudio de caso El Caribe. Eds.; R Rivera; K Fernández. Ediciones INCA. Cuba. 166 p.

Tawaraya, K; Hashimoto, K; Wagatsuma, T.1998. Effect of root exudate fractions from P-deficient and P-sufficient onion plants on root colonisation by the arbuscular mycorrhizal fungus Gigaspora margarita (disco compacto, 8 mm).Mycorrhiza 8 (2):67-70. Disponible en base de datos Teel, CD volumen 241.

Triana, JR. 2004. En la producción agrícola: Resultados obtenidos en diferentes modelos productivos. In Encuentro Mesoamericano y del Caribe (I), Encuentro Costarricense de Agricultores Experimentadores e Investigadores en Producción Orgánica (III, 2003,
Escuela Centroamericana de Ganadería). Eds. G Soto; P Descamps. San José, CR, Del Norte. p. 48.

Singh, S; Kapoor, KK. 1998. Effects of inoculation of phosphate-solubilizing microorganisms and an arbuscular mycorrhizal fungus on mungbean grown under natural soil conditions. Mycorrhiza 7 (5): 249-253. Disponible en base de datos Teel, CD volumen 241 .

Singh, HP; Singh, TA. 1993. The interaction of rockphosphate, Bradyrhizobium, vesicular-arbuscular mycorrhizae and phosphate-solubilizing microbes on soybean grown in a sub-Himalayan mollisol (disco compacto, $8 \mathrm{~mm}$ ). Mycorrhiza 4 (1):37-43. Disponible en base de datos Teel, CD volumen 30 .

Siquiera, JO; Saggin Jr, OJ; Flores Aylas, WW; Guimaraes P, TG. 1998. Arbuscular mycorrhizal inoculation and superphosphate application influence plant development and yield of coffee in Brazil (disco compacto, 8 mm). Mycorrhiza 7 (6): 293-300. Disponible en base de datos Teel, CD volumen 241.

Verma, RK; Arya, ID.1998. Effect of arbuscular mycorrhizal fungal isolates and organic manure on growth and mycorrhization of micropropagated Dendrocalamus asper plantlets and on spore production in their rhizosphere (disco compacto, $8 \mathrm{~mm}$ ). Mycorrhiza 8 (2):113-116. Disponible en base de datos Teel, CD volumen 241 .

Wang, B; Funakoshi, DM; Dalpe, Y; Hamel, C. 2002. Phosphorus-32 absorption and translocation to host plants by arbuscular mycorrhizal fungi at low root-zone temperatura (disco compacto, $8 \mathrm{~mm}$ ). Mycorrhiza 12 (2): 93-96. Disponible en base de datos Teel, CD volumen 383 . 
